\title{
Neural Predictors of Treatment Response in Depression
}

\author{
Catherine J. Harmer
}

Published online: 15 July 2014

(C) Springer International Publishing AG 2014

\begin{abstract}
Depression is a leading cause of disability and treatment response is highly variable between patients. The current review focuses on recent findings from studies exploring neural predictors of treatment response to conventional antidepressant drugs, psychological treatment, and novel candidate interventions. Structural markers of response have revealed that decreased volume of key substrates such as the hippocampus and orbitofrontal cortex are predictive of poor response to antidepressant drug treatment, and diffusion tensor imaging offers potentially relevant information for future studies. Pretreatment measures of rostral anterior cingulate activity (from functional magnetic resonance imaging- and electroencephalogram-based approaches) have also been highlighted as a biomarker for antidepressant drug response. The relative utility of measurements taken at baseline versus early change for refining treatment in individual patients is considered. The response to novel treatments such as scopolamine, ketamine, and deep brain stimulation also offer promise for understanding targets of treatment and predictors for tailoring treatment. There is considerable overlap between neural biomarkers for different treatments although positron emission tomography metabolism in the anterior insula may offer a differential predictor of response to pharmacotherapy versus psychotherapy in depression. These insights provide important information about the potential targets and mechanisms underlying the prediction of treatment effects in depression. Future work needs to explore these different predictors between treatment arms in prospectively defined studies to evaluate whether these markers may have utility in the clinic.
\end{abstract}

C. J. Harmer $(\bowtie)$

University Department of Psychiatry, Warneford Hospital, Oxford OX3 7JX, UK

e-mail: catherine.harmer@psych.ox.ac.uk
Keywords Depression · Antidepressants · Prediction · Markers $\cdot$ Treatment $\cdot$ Efficacy

\section{Introduction}

Depression is becoming increasingly recognized as a leading cause of disability worldwide [ $1 \bullet]$. Although there are a range of effective treatments, remission from depression is typically reached in less than half of all treated patients [2]. Persisting through adequate trials of two or more different treatments can increase overall likelihood of success in an individual [2]; however, this persistence is uncommon in real-world practice where each new treatment attempt can only be fully evaluated after a number of weeks. Faster matching of patients with effective treatments could therefore have dramatic effects on the outcome of major depression and its associated disability.

There is currently intense research interest in using pretreatment markers to predict treatment response in major depression, and several large multicenter studies are underway which include neural measures of function (e.g. the EMBARC [Establishing Moderators and Biosignatures of Antidepressant Response for Clinical Care] study). There are a number of ways in which these markers may be useful to refine clinical practice. First, these markers may provide a general measure of likely response and therefore be able to identify a subgroup of patients for whom standard treatments are unlikely to be effective and who may require additional interventions from an early stage of treatment. Second, and of potentially greater utility, these markers may provide a differential signal of likely response to different treatment modalities such as the likelihood of responding to psychological versus pharmacological treatments [3•]. Differential markers could also help tailor specific drug treatments with different mechanisms of action to individual patients; for example, those most likely to benefit from serotoninergic versus catecholamine agents. 
Although neuroimaging forms an expensive prognostic marker, if the signal is sufficiently robust then its use may still be cost effective. Equally, understanding the neural mechanisms related to treatment response or remission may help inform and refine simple behavioral or neuropsychological methods which hold promise and may be readily deployed in a clinical context. This review summarizes new findings from studies using neural predictors of treatment response in depression over the last year-what have we learnt and where do we need to go from here?

\section{Predicting Antidepressant Drug Response}

\section{Structural Markers}

Major depression has been associated with reduced brain volume across a network of areas, including the frontal cortex, orbitofrontal cortex, cingulate cortex, hippocampus, and striatum (for a recent meta-analysis see Arnone et al. [4]). Previous research also suggests that variability in brain morphometry can predict speed of response and likelihood of achieving remission following conventional antidepressant drug treatment, with reduced volume in hippocampus being the most consistent predictor of poor outcome (for a review see MacQueen [5]). A recent study reported that grey matter volume in the left hippocampal complex, posterior cingulate, right temporolateral cortex, and subcallosal cingulate was associated with a slower response to antidepressant treatment after 6 weeks in a relatively large sample $(n=167)$ of inpatients with mood disorder [6]. This was a naturalistic study and the majority of patients were already medicated at assessment. Analysis of the same dataset for remission rather than response led to no significant neural predictors, but treatment course was relatively short in this study and may therefore not have been powered to detect this association. In a longer study over 24 weeks, Ribeiz et al. [7] reported no association of hippocampal volume with remission in a largely unmedicated but relatively small sample of 30 patients with late-life depression (60 years or over). Despite this lack of prediction from hippocampal volume, reduced volume of the left superior orbitofrontal cortex was associated with poor outcome (defined as a Montgomery-Åsberg Depression Rating Scale [MADRS] score of 9 or more by week 24). A comparison with a healthy volunteer sample revealed that this patient group had reduced volume in bilateral orbitofrontal cortex but not the hippocampus at baseline.

Delorenzo et al. [8] explored the potential utility of diffusion tensor imaging (DTI) to predict response to selective serotonin reuptake inhibitor (SSRI) treatment in 18 patients with depression. Fractional anisotropy, as a measure of white matter tract integrity between the midbrain/raphe area (where serotonin cell bodies are located) and the amygdala and hippocampus was computed. Decreased fractional anisotropy between the midbrain and the amygdala was associated with non-remission, whereas fractional anisotropy in the hippocampal tract was not predictive of outcome. This study highlights the potential for DTI to provide relevant information for treatment response, perhaps in combination with other imaging methods commonly employed.

Together, these results suggest that structural markers of function may hold promise for the identification of patients likely to have a poor response to antidepressant drug treatment. This includes measures of volume in the hippocampus and orbitofrontal cortex as well as differences in white matter tract integrity between the raphe and amygdala. The identification of hippocampus volume as a potential predictor may be relevant given evidence that antidepressant treatment increases the volume of this area, although typically to a lesser extent than the effect of depression, i.e. it is only partially normalized with 8 weeks of treatment [9]. The role of this area for learning and memory may also be important for the translation of early changes in emotional processing into sustained improvement in mood and symptoms of depression [10].

However, the direct clinical application of these findings is still far from complete. There is no evidence that these markers provide differential information about optimal treatment for an individual, and while the connection between low hippocampal volume and poor response emerges relatively consistently across studies, this is not always replicated. Whether these differences are likely to have the necessary sufficient sensitivity and specificity to be taken forward clinically therefore needs further investigation in large-scale studies. Furthermore, the impact of targeting the identified subgroup of patients (e.g. those with low hippocampal volumes) using additional intervention early in treatment needs to be formally investigated to assess whether this prognostic marker can lead to improved treatment outcome in those for whom standard approaches are suboptimal.

\section{Functional Markers}

Functional markers of neural function can be examined using functional magnetic resonance imaging (fMRI), magnetoencephalography (MEG), and electroencephalogram (EEG), both in the resting state and in task-based designs. A metaanalysis of 23 studies revealed that pretreatment activity within the rostral anterior cingulate (rACC; Brodmann area 32 and inferior parts of 24) is a predictor of treatment response across treatments and imaging methods, with a weighted effect size of 0.9 [11•]. Specifically, increased baseline activity or response in this rACC area was associated with improved outcome in the treatment of depression. Thirteen of the studies showing a positive signal in this meta-analysis explored $\mathrm{rACC}$ function during the resting state, whereas the other six used task-based designs, suggesting some correspondence between 
different approaches to capture rACC function. It has also been noted that the prediction of response to pharmacotherapy versus psychotherapy for depression may be associated with a divergent pattern of perigenual ACC response across different studies [12]. In particular, increased ACC response to negative stimuli was predictive of better outcome with pharmacotherapy but worse outcome following psychological interventions [12]. However, these differences between treatments were noted in separate investigations using different paradigms, and therefore direct assessments in randomized designs are needed [12]. A number of studies using pretreatment neural measures of function in the prediction of outcome in depression have subsequently been reported over the last year.

\section{Pretreatment Predictors}

Using a baseline EEG measure, Rentzsch et al. [13] reported an association between slow frequency (delta) power in the right $\mathrm{ACC}$ and response after 4 weeks of antidepressant treatment in 31 inpatients with major depression. Delta-frequency power in this region predicted response with good sensitivity of $82 \%$ and specificity of $100 \%$, which was greater than the prediction provided by early (2-week) clinical response to treatment. Further assessment of this neural marker is therefore warranted in a larger independent sample.

Three studies by Jaworska et al. [14-16] have used EEG measures to predict treatment response in depressed patients randomized to receive escitalopram, bupropion, or their combination ( $n=51-53)$. In the first study, the loudness dependence of auditory evoked potentials (LDAEP) was assessed at baseline [14]. N1, P2, and N1/P2 LDAEP slopes were assessed as potential predictors of responders at week 12. Baseline N1 LDAEP and increased P2 LDAEP after week 1 of treatment were predictive of later clinical responses. These findings suggest that baseline auditory N1 is associated with intervention outcome but did not replicate previous work which supports baseline P2 in the LDAEP as a marker of subsequent improvement with treatment (e.g. [17] and [18]). Further work is therefore required to consider which component provides the most reliable information and whether this can withstand correction for multiple comparisons. A second report by the same group, based on the same patient cohort, explored the role of the auditory P3 in a novelty oddball task [15]. Non-responders to antidepressant treatment had smaller baseline $\mathrm{P} 3 \mathrm{a} / \mathrm{b}$ amplitudes than responders and a sample of healthy controls, although, again, multiple predictors were assessed. In both these studies, general predictors of outcome were identified which were not specific to the medication received despite the differences in neurochemical actions between escitalopram (an SSRI) and buproprion (a noradrenaline and dopamine reuptake inhibitor). The third study assessed resting frontal and posterior alpha 2 power and asymmetry as well as ACC localized theta [16]. Treatment response (across arms) was weakly predicted by frontal baseline alpha $_{2}$ power, with treatment responders exhibiting greater baseline alpha 2 . No overall prediction was provided by ACC theta or early (week 1) change in EEG measures. However, there were associations between early change in these measures and response as a function of treatment arm. These were complex small effects and require replication in a larger sample.

Hunter et al. [19] examined whether ACC activity at rest in EEG is a stable predictor of treatment response across time by collecting this measure just before and 5 weeks preceding antidepressant treatment. The relationship between rACC activity and clinical response to the SSRI sertraline was stronger when theta activity in the rACC level was measured just before the beginning of medication, compared with 5 weeks before. These results suggest that rACC activity may be a dynamic marker which is associated with treatment outcome rather than a stable or trait-like measure. This was a small study $(n=22)$ and subjects received placebo between the two measurements, which may have influenced responses. These effects are also difficult to disentangle since differences in symptoms or mood between the two assessments were not reported or included in the analysis. However, it does highlight the potential for this biomarker to be modified by nondrug manipulations, or over time, which may have relevance for improving clinical response if this marker is causally related to outcome.

Machine learning methodology can be applied to EEG data and may improve prediction by combining multiple sources of information. Khodayari-Rostamabad et al. [20] collected pretreatment resting state EEG data in 22 treatment-resistant patients prior to 6 weeks of SSRI administration (sertraline in most cases). Response was relatively low in this treatmentresistant sample and improvement was therefore defined by a $30 \%$ improvement or more in the Hamilton Depression Rating Scale (HAM-D), rather than the $50 \%$ cut-off used in most cited studies. The sensitivity of the machine learning algorithm was $94.9 \%$, and the specificity of prediction was $80.9 \%$. Again, replication in an independent large sample will be crucial.

Using fMRI during implicit face emotion processing, Victor et al. [21•] reported that pretreatment response in the pregenual cingulate to sad versus happy masked faces was correlated positively with clinical improvement during sertraline treatment in ten depressed patients. Activity in this same area was decreased following treatment, suggesting that the response of this area might be targeted (along with other parts of the limbic system; see Victor et al. [22]) following effective intervention. These results therefore add to the dataset highlighting the role of the rACC in predicting treatment response, and suggest that implicit face processing may be a useful paradigm to explore these effects. 
Another recent study used an emotion-based task as a predictor of treatment response to 8 weeks of escitalopram in 17 patients with depression [23], based on an overlapping patient sample to the DTI study reported above [8]. Lower blood oxygen level-dependent (BOLD) responses to negative stimuli across a large network (including the dorsolateral prefrontal cortex [DLPFC], subgenual ACC, insula, posterior cingulate, caudate, and thalamus) were correlated with improvement following subsequent antidepressant drug treatment. A similar prediction was offered by response to neutral and positive words, suggesting that these effects may not be valence-specific but perhaps more generally involved in selfreferential processing or cognitive control. Consistent with this, the paradigm involved the presentation of stimuli which were partly generated by the patients as personally relevant. Replication in a large sample is needed to assess the role and prediction provided by baseline responses in emotional processing fMRI, as well as more detailed examination of the specific subregions of the ACC which are predictive of treatment response.

Miller et al. [24] also tested whether baseline 5-HT $1 \mathrm{~A}$ receptor binding (measured with positron emission tomography [PET]) is associated with remission following escitalopram treatment in a partly overlapping depressed sample. Remitters were found to have higher $5-\mathrm{HT}_{1 \mathrm{~A}}$ binding in the raphe nuclei compared with non-remitters, although there were no differences in neural areas known to be innervated by serotonin neurones. This finding was interpreted in line with the idea that SSRIs may be most effective in those with increased $5-\mathrm{HT}_{1 \mathrm{~A}}$ autoreceptor function, who are most likely to experience increased serotonin efflux once these receptors are desensitized as part of repeated SSRI treatment [23]. These effects did not correlate with the DTI findings in the same sample above [8], suggesting they may tap into separate aspects of function. Again, these results have relevance for mechanisms of action of SSRI treatment but are not sufficiently powerful with current methods to predict responses in individual patients. It will also be interesting to observe whether these effects are specific to SSRI drug treatments or are also seen as a general marker of response.

\section{Early Change During Treatment}

While baseline predictors may be simpler to employ in clinical practice, the early change in neural function induced by treatment also offers certain advantages. Contemporary theories of antidepressant drug action suggest that early change in emotional processing is a mechanism of later therapeutic response in depression and may therefore represent a marker of early response $[10,25]$. This approach also has the potential to provide an early biomarker which is relevant to the specific medication taken by the individual, i.e. the ability of a specific agent to shift aberrant processing can be tested in each patient, leading to the potential to change treatment earlier than in standard clinical practice. This could then provide a signal that is specific to that agent at that time in that individual at a particular dose, rather than providing a general marker of outcome.

Early changes in frontal EEG signal have previously been related to clinical response to antidepressant drug treatment $[26,27]$. In particular, change in prefrontal theta band cordance after 1 week of treatment has been shown across studies to predict response and remission in depression. In a recent study, Bares et al. [28] reported that this measure was predictive of response to both DLPFC repetitive transcranial magnetic stimulation (rTMS) and venlafaxine in a total of 50 patients with major depression. These results suggest that the ability of an intervention to shift the prefrontal signal may be important across treatment approaches. A related EEG measure that uses a reduced electrode montage, focused on the prefrontal area, and integrates information on alpha and theta band activity may also hold predictive promise [26]. In a recent publication, Cook et al. [29] used this frontal EEG marker taken at baseline and after 1 week of escitalopram treatment as a predictor of speed to remission and sustained remission in the BRITE-MD (Biomarkers for Rapid Identification of Treatment Effectiveness in Major Depression) dataset [30]. This EEG biomarker was predictive of both time to remission and sustained remission by week 13 . These approaches are based on a standardized measure, specified in advance and consistent across studies. The level of sensitivity attained (i.e. $52.8 \%$ for prediction of week 13 remission) is relevant for our understanding of mechanisms of action, but may still need to be optimized before it can be used reliably to inform clinical decision making.

Together, this work suggests that rACC activity is a biomarker for likely response to a range of different treatments. Frontal signal measured with EEG (believed to measure ACC responses, albeit less specifically) also offers promise for understanding the mechanisms important for treatment response. EEG may be more practical and cost effective for clinical deployment than fMRI, although the sensitivity and selectivity of this prediction may need further optimization. Future studies may wish to consider whether the improved localization offered by MEG enhances the prediction of treatment outcome in depression. This may be particularly relevant in light of evidence that different subregions of the ACC can show opposing predictions of treatment outcome (e.g. McGrath et al. [31]), but which may be difficult to disambiguate with EEG alone.The relative richness of information provided by baseline predictors versus early change following treatment will also be important to evaluate. Baseline predictors may give a measure of the likely chance of responding across treatments, whereas early change will provide a measure of response to the specific agents being taken by a patient. The role of prediction from activity in the rACC and frontal 
EEG signal are often interpreted as reflecting the function of the default mode network. Future work is needed to assess whether manipulations designed to modulate DMN function may have a role in improving treatment outcome.

\section{Novel Candidate Antidepressant Treatments}

There has been recent interest in the use of novel and fastacting interventions for the treatment of depression, including both scopolamine (a muscarinic antagonist) and ketamine (an NMDA antagonist) in treatment-resistant depression [32•]. Ketamine and scopolamine have been shown to have antidepressant effects which emerge in hours to days rather than weeks, making them intriguing candidates for exploring prediction of effects [32]. Furey et al. [33] examined the prediction of the rapid antidepressant effect of scopolamine using fMRI during a face identity and face emotion working memory task in 15 depressed patients. Baseline BOLD response in the bilateral middle occipital cortex, specifically during emotion working memory, correlated with treatment response magnitude (percentage change in MADRS). Specifically, those patients with decreased occipital response during the emotion working memory task were most likely to show a therapeutic response to the drug. Responses in this area tended to be lower in patients with major depression compared with matched healthy controls, suggesting that patients most likely to benefit are those with the largest response deficit in this area. Assessment of the same task after scopolamine treatment revealed enhanced responses in this occipital area, supporting a remediation of this difference as a function of treatment. Differences in occipital responses to emotional stimuli may be associated with changes in visual attention or engagement with the task and these results implicate a potential biomarker for patients who are likely to respond to this novel candidate treatment.

Nugent et al. [34] explored the prediction of clinical response in depressed patients with bipolar disorder using FDGPET following a single intravenous infusion of ketamine. All patients were maintained on lithium or sodium valporate during the study. Improvement in depression was associated with increased metabolic increases after ketamine compared with placebo. In addition, metabolism in the subgenual ACC following placebo was positively correlated with improved depression following ketamine. Although examined under placebo, this appears to parallel findings highlighting baseline ACC activity as predictive of therapeutic benefit with antidepressant treatment [11•], and may represent a common neurobiological correlate across successful treatment approaches.

The subcallosal part of the ACC (BA 25 and parts of 24 and 32 ) has recently received intense research interest as a location for deep brain stimulation (DBS) in depression (see Hamani et al. [35] for a review). This site was selected since activity in this area correlated with the induction of sadness in volunteers and was sensitive to antidepressant drug administration in depression (see Hamani et al. [35]). Antidepressant effects with chronic DBS of the subcallosal ACC have been reported across a number of studies [35]; however, there is variability in patient response to this treatment and its invasive nature highlights the particular importance of identifying biomarkers which can be used to refine the use of DBS. A recent study by Broadway et al. [36] investigated pretreatment and early treatment EEG markers of response in 12 patients undergoing DBS for depression. Six were classified as responders after 24 weeks of DBS and six were non-responders. Reduced frontal theta cordance at baseline and increased cordance after 4 weeks of treatment was predictive of improved clinical response at 24 weeks. The direction of these predictive markers are opposite to those commonly reported with antidepressant drugs, i.e. where higher frontal theta cordance at baseline are typically predictive of a better response. These differences may relate to small methodological differences in EEG recording, or that patients in this study were treatment resistant and already taking antidepressant medication at baseline. Alternatively, it remains a possibility that this pattern may represent a signal for patients unlikely to respond to conventional medications but who may benefit from direct stimulation of the subcallosal cingulate. Replication in a larger sample is needed to evaluate the robustness of these effects.

\section{Repetitive Transcranial Magnetic Stimulation}

DLPFC rTMS appears to have clinical utility in the treatment of depression [37], although the optimal parameters for efficacy still need to be fully determined. A study reviewed above suggests that frontal cordance with EEG after 1 week of right frontal (1hz) rTMS predicted clinical response similar to that seen for venlafaxine [28]. Arns et al. [38] also used an EEG measure based on three different non-linear metrics at baseline as a predictor of response to rTMS (either left DLPFC $10 \mathrm{~Hz}$ or right DLPFC $1 \mathrm{~Hz}$ ). This was applied concurrently with psychotherapy, and $32 \%$ of patients were also receiving medication. The averaged non-linear measures did not yield prognostic value; however, the change of one metric (LempelZiv Complexity) from 1-min to another yielded a prediction for non-response. Thus, patients unlikely to respond showed reduced complexity from minute 1-2 compared with healthy controls and the depressed group who subsequently responded to the treatment.

By contrast, a study by Widge et al. [39] showed no significant predictors of response to 6 weeks left DLPFC $(10 \mathrm{~Hz})$ rTMS treatment in 180 unmedicated patients using EEG measures. This involved correlation with EEG power in multiple bands as well as early 1-week change in EEG 
response (as defined above; see Cook et al. [27]), but not the non-linear methods described by Arns et al. [38]. This represents a large-scale study in an independent research group, and therefore is a cautionary note for the ability to predict rTMS efficacy from baseline and early changes in EEG.

Differences in prediction of rTMS treatment effects in depression are seen between studies. There is also considerable variability in how rTMS is applied, including the laterality of stimulation and associated frequency as well as its combination with other treatments. We therefore need further studies across treatment arms to explore whether similar or distinct predictors are seen for this intervention for depression.

\section{Psychological Treatment}

Response to 12 weeks of cognitive behavioral therapy (CBT) in older adults has been examined by Thompson et al. [40] in a largely medicated sample. The neural response during the Wisconsin Card Sorting Task at baseline was used as a predictor of subsequent remission. Decreased activation in the left inferior frontal cortex and right superior frontal gyrus, as well as increased activity in the right middle frontal gyrus and left superior frontal gyrus, predicted a positive response to CBT. These findings may indicate that patients with intact executive function are most likely to benefit from CBT in late- life depression. This is consistent with another study in a younger sample of patients with post-traumatic stress disorder, which reported that increased function of neural circuitry supporting executive function was predictive of CBT efficacy [41].

Early predictors of response to psychological intervention are needed; however, it is particularly important to consider markers that can provide a differential signal of whether psychological versus pharmacological treatment is most likely to benefit a particular patient.

\section{Drug versus Psychological Treatments}

There is a scarcity of studies directly contrasting predictors of different treatments in randomized designs. In a landmark study, McGrath et al. [3•] examined the ability of pretreatment brain glucose metabolism measured by PET to predict remission in patients with major depressive disorder randomized to receive an SSRI (escitalopram, $n=39)$ or CBT $(n=41)$. The results support pretreatment differential prediction from a network of areas, including the right anterior insula, right inferior temporal cortex, left amygdala, left premotor cortex, right motor cortex, and precuneus. Of these, the signal in the anterior insula best discriminated treatment outcome; insula hypometabolism was associated with subsequent remission to CBT but poor response to escitalopram, whereas insula

\section{Decreased response to antidepressant drug treatments}

\section{Method used}

Observed predictor

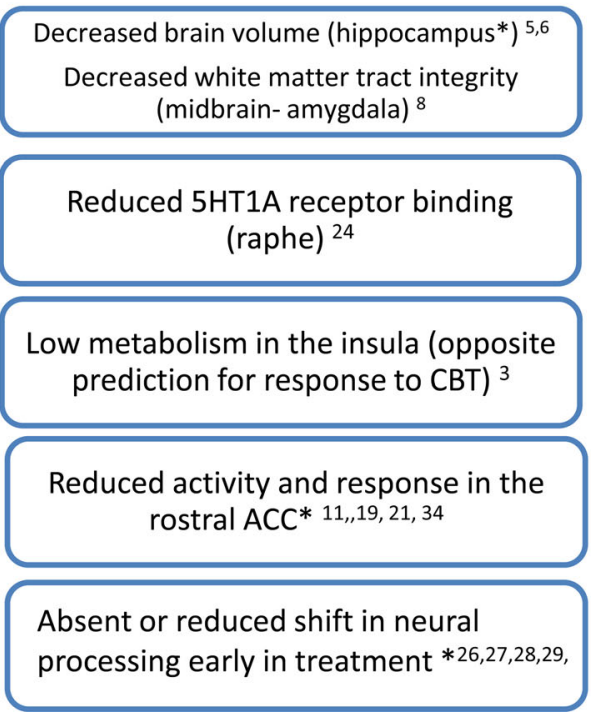

Structural: MRI/DTI

Receptor binding: PET

Functional: resting PET

Functional: resting and task based fMRI; EEG

Functional: resting EEG
Fig. 1 Recent studies support a role for neural markers to predict the level of treatment response to antidepressant drugs in depression. Example observations associated with reduced response or non-remission to antidepressants in depression are featured. Citations for the relevant featured studies are noted in number form. An asterisk represents observations reported across multiple studies. $M R I$ magnetic resonance imaging, DTI diffusion tensor imaging, PET positron emission tomography, $f M R I$ functional magnetic resonance imaging, $E E G$ electroencephalogram, $C B T$ cognitive behavioral therapy, $A C C$ anterior cingulate 
hypermetabolism was associated with remission to escitalopram but poor response to CBT. The rACC did not discriminate between remitters and non-remitters, although there was a non-signifcant prediction of response in the escitalopram group in the post hoc analysis. These results suggest that there may be categorical differences in neural markers of patients who require CBT versus those who require SSRI treatment. Pending a prospective study using metabolism in the anterior insula to guide treatment allocation, this is an exciting result which may help inform clinical practice. PET is one of the most expensive and difficult to use neuroimaging methods and therefore extending the same approach to either resting state fMRI or behavioral measures which are linked to insula arousal (such as interoceptive awareness measures) will be important.

A further publication based on the same study explored PET predictors of poor outcome [31]. Here, data included a second experimental phase where non-remitters were treated with both CBT and the SSRI. Pretreatment activity in the subcallosal ACC was higher in the non-responders to either phase of treatment compared with remitters. Although this subcallosal area of the cingulate is often considered part of the more general rACC, there is evidence that the rACC and subcallosal ACC differ in terms of anatomical connectivity, cytology, and neurotransmitter receptor organization [42, 43], making this division an important consideration for future studies. In the whole-brain analysis, increased temporal sulcus activity was also associated with non-response to both treatments. There were no clinical predictors that were able to differentiate between these subgroups, suggesting added value for neural markers of function; however, the subgroup analyses were small in this study (9 non-responders to both CBT and escitalopram vs. 12 combined remitters). Further clinical studies exploring whether earlier targeting of a subgroup based on pretreatment neural markers (i.e. those with increased subcallosal ACC activity) would improve overall clinical outcome are needed.

\section{Conclusions}

The ability to predict treatment response in depression to different treatments would transform clinical practice. There is evidence that both pretreatment and early change markers of neural function hold promise (see Fig. 1). Decreased volume of critical areas involved in depression, such as the hippocampus, appear to be predictive of poor outcome with conventional treatments. Furthermore, decreased activity in the rostral ACC and reduced change in frontal EEG signal early in treatment are also markers of non-response and poor outcome. These predictors are seen across many different treatment approaches, although preliminary work suggests that an opposing pattern may predict response to subcallosal ACC DBS. Recent studies have also highlighted multiple novel measures which may hold promise for the prediction of treatment response. Replication of these indices is needed as well as studies evaluating whether these predictors can usefully be combined using machine learning algorithms. This approach may provide greater predictive power and be able to provide a condensed source of information for clinical decision making.

The early ability of a specific treatment to shift aberrant neural patterns of response is also a marker of the ability of that same treatment to be therapeutic in an individual. This has the potential for greater specificity in that it measures a response to a particular treatment, at a given time and dose, and may therefore be used to refine clinical decision making earlier than is typically possible. Further work is needed to assess whether using these kinds of early markers in clinical practice improves overall outcome and whether including these neural measures are cost effective by limiting the length of disability associated with this disorder. There is also an urgent need for these findings to be replicated in large-scale studies with multiple treatment arms to allow direct comparisons between predictors to different interventions.

There is evidence that distinct neural markers may predict remission to drug versus psychological treatment using PET neuroimaging. This exciting discovery paves the way for future larger scale prospective treatment studies.

\section{Compliance with Ethics Guidelines}

Conflict of Interest Catherine Harmer has served as a consultant for Lunbeck, P1Vital, Eli Lilly, and Servier. She has also received research grants from Johnson and Johnson, Lundbeck, Sunovion, Astra-Zeneca and UCB, and has stock options in Oxford Psychologists Ltd.

Human and Animal Rights and Informed Consent This article does not contain any studies with human or animal subjects performed by the authors.

\section{References}

Papers of particular interest, published recently, have been highlighted as:

- Of importance

1. Ferrari AJ, Charlson FJ, Norman RE, Patten SB, Freedman G, Murray CJ, et al. Burden of depressive disorders by country, sex, age, and year: findings from the global burden of disease study 2010. PLoS Med. 2013;10(11):e1001547. A clear summary of the worldwide disability caused by depression and the need for effective intervention.

2. Rush AJ, Trivedi MH, Wisniewski SR, Nierenberg AA, Stewart JW, Warden D, et al. Acute and longer-term outcomes in depressed outpatients requiring one or several treatment steps: a STAR*D report. Am J Psychiatry. 2006;163:1905-17. 
3. McGrath CL, Kelley ME, Holtzheimer PE, Dunlop BW, Craighead WE, Franco AR, et al. Toward a neuroimaging treatment selection biomarker for major depressive disorder. JAMA Psychiatr. 2013;70:821-9. The first study to use a randomised controlled study to explore baseline PET predictors of remission to pharmacotherapy versus psychological treatment in depression. The results suggest that metabolism of the insula provides a differential marker for remission with drug versus psychological treatment.

4. Arnone D, McIntosh AM, Ebmeier KP, Munafò MR, Anderson IM. Magnetic resonance imaging studies in unipolar depression: systematic review and meta-regression analyses. Eur Neuropsychopharmacol. 2012;22:1-16.

5. MacQueen GM. Magnetic resonance imaging and prediction of outcome in patients with major depressive disorder. J Psychiatry Neurosci. 2009;34:343-9.

6. Sämann PG, Höhn D, Chechko N, Kloiber S, Lucae S, Ising M, et al. Prediction of antidepressant treatment response from gray matter volume across diagnostic categories. Eur Neuropsychopharmacol. 2013;23:1503-15.

7. Ribeiz SR, Duran F, Oliveira MC, Bezerra D, Castro CC, Steffens $\mathrm{DC}$, et al. Structural brain changes as biomarkers and outcome predictors in patients with late-life depression: a cross-sectional and prospective study. PLoS One. 2013;8:e80049.

8. Delorenzo C, Delaparte L, Thapa-Chhetry B, Miller JM, Mann JJ, Parsey RV. Prediction of selective serotonin reuptake inhibitor response using diffusion-weighted MRI. Front Psychiatr. 2013;4:5.

9. Arnone D, McKie S, Elliott R, Juhasz G, Thomas EJ, Downey D, et al. State-dependent changes in hippocampal grey matter in depression. Mol Psychiatry. 2013;18:1265-72.

10. Harmer CJ, Goodwin GM, Cowen PJ. Why do antidepressants take so long to work? A cognitive neuropsychological model of antidepressant drug action. Br J Psychiatry. 2009;195:102-8.

11. Pizzagalli DA. Frontocingulate dysfunction in depression: toward biomarkers of treatment response. Neuropsychopharmacology. 2011;36:183-206. A meta-analysis highlighting the importance of activity and response in the $\mathrm{rACC}$ as a predictor of treatment success in depression. This association was seen across imaging methods, paradigms and treatment type.

12. Roiser JP, Elliott R, Sahakian BJ. Cognitive mechanisms of treatment in depression. Neuropsychopharmacology. 2012;37:117-36.

13. Rentzsch J, Adli M, Wiethoff K, Gómez-Carrillo de Castro A, Gallinat J. Pretreatment anterior cingulate activity predicts antidepressant treatment response in major depressive episodes. Eur Arch Psychiatry Clin Neurosci. 2014;264:213-23.

14. Jaworska N, Blondeau C, Tessier P, Norris S, Fusee W, Blier P, et al. Response prediction to antidepressants using scalp and sourcelocalized loudness dependence of auditory evoked potential (LDAEP) slopes. Prog Neuropsychopharmacol Biol Psychiatry. 2013;44:100-7.

15. Jaworska N, De Somma E, Blondeau C, Tessier P, Norris S, Fusee W, et al. Auditory P3 in antidepressant pharmacotherapy treatment responders, non-responders and controls. Eur Neuropsychopharmacol. 2013;23:1561-9.

16. Jaworska N, Blondeau C, Tessier P, Norris S, Fusee W, Blier P, et al. Examining relations between alpha power as well as anterior cingulate cortex-localized theta activity and response to single or dual antidepressant pharmacotherapies. J Psychopharmacol. 2014;28(6): 587-95.

17. Gallinat J, Bottlender R, Juckel G, Munke-Puchner A, Stotz G, Kuss HJ, et al. The loudness dependency of the auditory evoked N1/P2-component as a predictor of the acute SSRI response in depression. Psychopharmacology (Berl). 2000;148:404-11.

18. Mulert C, Juckel G, Augustin H, Hegerl U. Comparison between the analysis of the loudness dependency of the auditory N1/P2 component with LORETA and dipole source analysis in the prediction of treatment response to the selective serotonin reuptake inhibitor citalopram in major depression. Clin Neurophysiol. 2002;113:1566-72.

19. Hunter AM, Korb AS, Cook IA, Leuchter AF. Rostral anterior cingulate activity in major depressive disorder: state or trait marker of responsiveness to medication? J Neuropsychiatry Clin Neurosci. 2013;25:126-33.

20. Khodayari-Rostamabad A, Reilly JP, Hasey GM, de Bruin H, Maccrimmon DJ. A machine learning approach using EEG data to predict response to SSRI treatment for major depressive disorder. Clin Neurophysiol. 2013;124:1975-85.

21. Victor TA, Furey ML, Fromm SJ, Ohman A, Drevets WC. Changes in the neural correlates of implicit emotional face processing during antidepressant treatment in major depressive disorder. Int J Neuropsychopharmacol. 2013;16:2195-208. A study exploring responses to subliminal presentations of emotional facial expressions as a marker and predictor of treatment action in depression. Here, baseline reactivity to sad versus happy facial expressions in the rostral ACC was predictive of response to the SSRI sertraline. This forms a subanalysis of a wider study assessing the effects of sertraline in the treatment of depressed patients [see 21].

22. Victor TA, Furey ML, Fromm SJ, Ohman A, Drevets WC. Relationship between amygdala responses to masked faces and mood state and treatment in major depressive disorder. Arch Gen Psychiatry. 2010;67:1128-38.

23. Miller JM, Schneck N, Siegle GJ, Chen Y, Ogden RT, Kikuchi T, et al. fMRI response to negative words and SSRI treatment outcome in major depressive disorder: a preliminary study. Psychiatry Res. 2013;214(3):296-305.

24. Miller JM, Hesselgrave N, Ogden RT, Zanderigo F, Oquendo MA, Mann JJ, et al. Brain serotonin 1A receptor binding as a predictor of treatment outcome in major depressive disorder. Biol Psychiatry. 2013;74(10):760-7.

25. Pringle A, Browning M, Cowen PJ, Harmer CJ. A cognitive neuropsychological model of antidepressant drug action. Prog Neuropsychopharmacol Biol Psychiatry. 2011;35:1586-92.

26. Leuchter AF, Cook IA, Hunter AM, Korb AS. A new paradigm for the prediction of antidepressant treatment response. Dialogues Clin Neurosci. 2009; 11:435-46.

27. Cook IA, Hunter AM, Korb AS, Leuchter AF. Do prefrontal midline electrodes provide unique neurophysiologic information in major depressive disorder? J Psychiatr Res. 2014;53:69-75.

28. Bares M, Brunovsky M, Novak T, Kopecek M, Stopkova P, Sos P, et al. QEEG theta cordance in the prediction of treatment outcome to prefrontal repetitive transcranial magnetic stimulation or venlafaxine ER in patients with major depressive disorder. Clin EEG Neurosci. Epub 7 Apr 2014.

29. Cook IA, Hunter AM, Gilmer WS, Iosifescu DV, Zisook S, Burgoyne KS, et al. Quantitative electroencephalogram biomarkers for predicting likelihood and speed of achieving sustained remission in major depression: a report from the biomarkers for rapid identification of treatment effectiveness in major depression (BRITE-MD) trial. J Clin Psychiatry. 2013;74:51-6.

30. Leuchter AF, Cook IA, Marangell LB, Gilmer WS, Burgoyne KS, Howland RH, et al. Comparative effectiveness of biomarkers and clinical indicators for predicting outcomes of SSRI treatment in major depressive disorder: results of the BRITE-MD study. Psychiatry Res. 2009;169:124-31.

31. McGrath CL, Kelley ME, Dunlop BW, Holtzheimer Iii PE, Craighead WE, Mayberg HS. Pretreatment brain states identify likely nonresponse to standard treatments for depression. Biol Psychiatry. Epub 19 Dec 2013.

32. Zarate C, Duman RS, Liu G, Sartori S, Quiroz J, Murck H. New paradigms for treatment-resistant depression. Ann N Y Acad Sci. 2013;1292:21-31. An excellent review of novel interventions for depression, including the use of fast-acting agents targeting neural plasticity. 
33. Furey ML, Drevets WC, Hoffman EM, Frankel E, Speer AM, Zarate Jr CA. Potential of pretreatment neural activity in the visual cortex during emotional processing to predict treatment response to scopolamine in major depressive disorder. JAMA Psychiatry. 2013;70:280-90.

34. Nugent AC, Diazgranados N, Carlson PJ, Ibrahim L, Luckenbaugh DA, Brutsche N, et al. Neural correlates of rapid antidepressant response to ketamine in bipolar disorder. Bipolar Disord. 2014;16: 119-28.

35. Hamani C, Mayberg H, Stone S, Laxton A, Haber S, Lozano AM. The subcallosal cingulate gyrus in the context of major depression. Biol Psychiatry. 2011;69(4):301-8.

36. Broadway JM, Holtzheimer PE, Hilimire MR, Parks NA, Devylder JE, Mayberg HS, et al. Frontal theta cordance predicts 6-month antidepressant response to subcallosal cingulate deep brain stimulation for treatment-resistant depression: a pilot study. Neuropsychopharmacology. 2012;37:1764-72.

37. Herrmann LL, Ebmeier KP. Factors modifying the efficacy of transcranial magnetic stimulation in the treatment of depression: a review. J Clin Psychiatry. 2006;67:1870-6.

38. Arns M, Cerquera A, Gutiérrez RM, Hasselman F, Freund JA. Non-linear EEG analyses predict non-response to rTMS treatment in major depressive disorder. Clin Neurophysiol. 2014;125(7):1392-9.

39. Widge AS, Avery DH, Zarkowski P. Baseline and treatmentemergent EEG biomarkers of antidepressant medication response do not predict response to repetitive transcranial magnetic stimulation. Brain Stimul. 2013;6:929-31.

40. Thompson DG, Kesler SR, Sudheimer K, Mehta KM, Thompson LW, Marquett RM, et al. fMRI activation during executive function predicts response to cognitive behavioral therapy in older, depressed adults. Am J Geriatr Psychiatry. Epub 18 Feb 2014.

41. Falconer E, Allen A, Felmingham KL, Williams LM, Bryant RA. Inhibitory neural activity predicts response to cognitive-behavioral therapy for posttraumatic stress disorder. J Clin Psychiatry. 2013;74:895-901.

42. Johansen-Berg H, Gutman DA, Behrens TE, Matthews PM, Rushworth MF, Katz E, et al. Anatomical connectivity of the subgenualcingulate region targeted with deep brain stimulation for treatment-resistant depression. Cereb Cortex. 2008;18:1374 83.

43. Palomero-Gallagher N, Mohlberg H, Zilles K, Vogt B. Cytology and receptor architecture of human anterior cingulate cortex. J Comp Neurol. 2008;508(6):906-26. 